\title{
Dedo Azul Não-Isquémico Espontâneo: Um Fenómeno Raro e Benigno
}

\author{
Spontaneous Non Ischaemic Blue Finger: A Rare and Benign Phenomenon
}

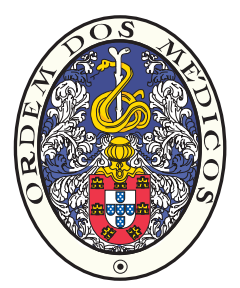

\author{
Daniela FRANCO $\rrbracket^{1}$, Daniela ALVES ${ }^{1}$, Ana Cristina ALMEIDA¹, Carlos Costa ALMEIDA², Cecília MORENO, \\ João FREIXO1 \\ Acta Med Port 2015 Jul-Aug;28(4):528-530
}

\section{RESUMO}

O dedo azul não-isquémico espontâneo é uma entidade rara e benigna, caracterizada pela alteração da coloração isolada de um dedo, com aparecimento e resolução espontânea. Este artigo relata o caso de uma mulher de 88 anos, que após algumas horas de permanência no serviço de urgência desenvolveu, sem trauma associado uma coloração violácea do terceiro dedo da mão direita, com pulsos mantidos, sem alteração da temperatura e indolor. A pesquisa etiológica realizada foi negativa. A doente foi reavaliada uma semana após o evento e mostrava regressão total da lesão. Apesar de existirem várias patologias que partilham sinais e sintomas semelhantes, o diagnóstico baseia-se no aparecimento espontâneo, na coloração violácea característica que poupa a polpa do dedo, e na resolução rápida sem necessidade de tratamento. Mesmo sendo um fenómeno benigno necessita de avaliação precoce para que o diagnóstico diferencial com patologias mais graves seja feito atempadamente.

Palavras-chave: Alterações da Pigmentação; Dedos; Isquémia.

\section{ABSTRACT}

The spontaneous non-ischaemic blue finger is a rare and benign disorder, characterized by purple discoloration of a finger, with complete resolution. This article reports the case of a woman of 88 years, which after a few hours of stay in the emergency department developed without associated trauma, a purplish color of the $3^{\text {rd }}$ finger of the right hand, with a palpable pulse and without temperature changes or pain. The etiological investigation was negative. The patient was assessed one week after the event and showed complete resolution. There are several diseases that share the same signs and symptoms, as such the diagnosis is based on the spontaneous violaceous color sparing the finger tip, and fast resolution without treatment. Though being a harmless phenomenon, it requires early assessment for timely differential diagnosis with severe pathologies.

Keywords: Fingers; Ischemia; Pigmentation Disorders.

\section{INTRODUÇÃo}

O dedo-azul não-isquémico espontâneo é uma condição rara, benigna e não dolorosa, que se apresenta com coloração violácea de um dedo isolado, ${ }^{1}$ ocorre mais frequentemente em mulheres de meia-idade e pode ser recorrente. ${ }^{2}$

Nestes casos a coloração do dedo tem uma tonalidade violácea e poupa na grande maioria dos casos sua a polpa. ${ }^{3,4}$

A sua resolução, tal como o aparecimento, ocorre de forma rápida e espontânea e sem necessidade de qualquer tratamento. ${ }^{5}$

Existem várias patologias que compartilham sinais $\mathrm{e}$ sintomas semelhantes, entre elas o fenómeno de Raynaud, a trombose digital espontânea, o síndrome Gardner-Diamond, a doença de Buerger, a isquemia, a libertação de micro-êmbolos ou o trauma. ${ }^{5,6}$

O diagnóstico diferencial com estas entidades é importante, pois a necessidade de tratamento e o prognóstico diferem imenso entre elas.

\section{CASO CLÍNICO}

Mulher de 88 anos, dependente nas atividades de vida diária desde há cinco anos, recorreu ao serviço de urgência (SU) por tosse com expetoração mucopurulenta com sete dias de evolução. Foi diagnosticada uma pneumonia adquirida na comunidade. Tratava-se de uma doente com hipertensão arterial, dislipidemia e doença de Alzheimer. Medicada com captopril $25 \mathrm{mg}$, sinvastatina $20 \mathrm{mg}$ e memantina $20 \mathrm{mg}$.

No momento da alta (após seis horas de permanência no SU) notou-se uma coloração violácea do terceiro dedo da mão direita de aparecimento súbito, sem trauma associado e indolor. À observação encontrava-se normotensa, a pele tinha temperatura normal, com pulsos cubital e radial mantidos e simétricos e sem alterações da sensibilidade ou da mobilidade. (Fig.s 1A and 1B)

Neste contexto a doente realizou radiografia da mão, que não mostrou qualquer alteração de relevo. Os exames analíticos realizados também não mostraram alterações importantes.

O diagnóstico de dedo azul não-isquémico espontâneo foi proposto, e a doente permaneceu no serviço de observação por 24 horas, durante as quais não houve agravamento da lesão nem aparecimento de novos sinais ou sintomas, tendo alta para o domicílio medicada para a pneumonia.

Ao fim de uma semana foi reavaliada em consulta externa. O estudo analítico para diagnóstico diferencial incluindo patologia auto-imune (lúpus eritematoso sistémico,

1. Serviço de Medicina Interna. Unidade Local de Saúde de Castelo Branco. Castelo Branco. Portugal.

2. Serviço de Cirurgia. Unidade Local de Saúde de Castelo Branco. Castelo Branco. Portugal.

$\triangle$ Autor correspondente: Daniela Franco. danielagouveiafranco@gmail.com

Recebido: 28 de Setembro de 2014 - Aceite: 20 de Maio de 2015 | Copyright @ Ordem dos Médicos 2015 

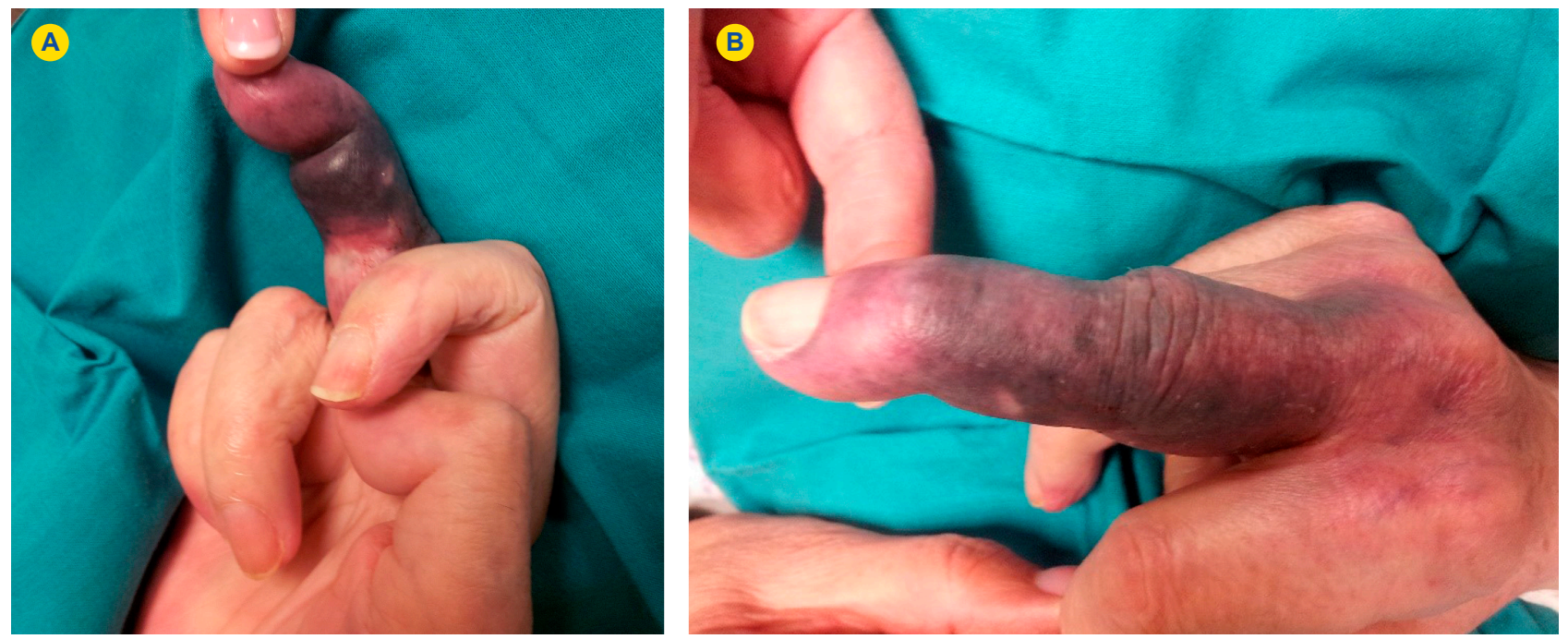

Figura 1 - Coloração violácea de aparecimento súbito e espontâneo do $3^{\circ}$ dedo da mão direita

síndrome Sjögren, doença mista do tecido conjuntivo, poli/ dermatomiosite, esclerodermia, artrite reumatóide e síndrome antifosfolípido) e trombofilias (factor $\mathrm{V}$ de Leiden, protrombina $20210 \mathrm{G} / \mathrm{A}, \mathrm{MTHFR}$, homocisteína, proteína C e $\mathrm{S}$, resistência à proteína $\mathrm{C}$ e anti-trombina III) foi negativo. A doente apresentava-se sem queixas álgicas, com regressão total da coloração violácea do dedo, e sem comprometimento funcional. (Fig. 2)

\section{DISCUSSÃO}

O diagnóstico de dedo azul não-isquémico espontâneo é um diagnóstico de exclusão. Antes de o assumir, é importante o diagnóstico diferencial com doenças mais graves e com prognóstico mais reservado, nomeadamente as de causa trombótica, embólica e auto-imune.

Entre as patologias com as quais é mandatório o diagnóstico diferencial destaca-se, o fenómeno de Raynaud, a trombose digital espontânea e a isquemia.

O fenómeno de Raynaud tende a aparecer em idade precoce, associa-se a doenças do tecido conjuntivo, ${ }^{6} \mathrm{e}$ apresenta frequentemente alterações progressivas da coloração. No caso apresentado, o estudo de doenças do te-

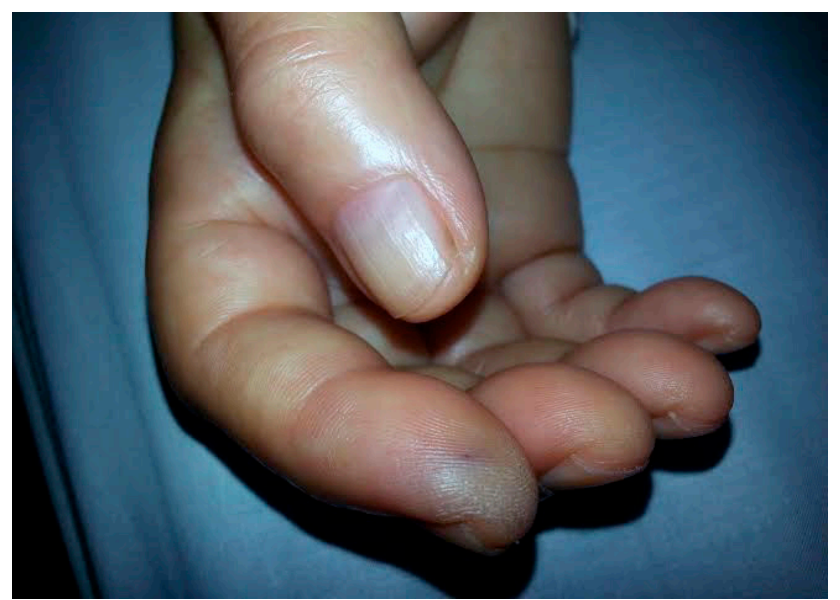

Figura 2 - Regressão total e espontânea da coloração violácea do $3^{\circ}$ dedo ao fim de uma semana cido conjuntivo foi negativo, e a evolução da coloração não foi a descrita.

Os doentes com trombose digital espontânea geralmente apresentam uma coloração menos exuberante, associada a nódulos palpáveis, sendo a terapêutica médica e por vezes cirúrgica essencial.

Por fim, na isquemia a coloração é mais arroxeada, os pulsos periféricos estão ausentes, ${ }^{6}$ existe diminuição da temperatura e compromisso da função, e implica tratamento. Aspetos que no caso relatado não estavam presentes e que permitiram excluir este diagnóstico.

A chave para o diagnóstico do dedo azul não-isquémico espontâneo, assenta na forma de aparecimento, na coloração violácea e no facto de poupar na maioria dos casos a polpa do dedo. ${ }^{5,6}$ A sua resolução rápida, sem passar pelos estádios clássicos de reabsorção de hematoma, e sem necessidade de qualquer tratamento, são também aspetos bastante sugestivos. ${ }^{6}$ Estas características foram evidentes no caso clínico apresentado.

\section{CONCLUSÃO}

O conhecimento e reconhecimento desta entidade é crucial, pois apesar de benigna, pode trazer grande preocupação, quer ao doente quer ao médico, pelo seu diagnóstico diferencial com doenças graves de origem trombótica, embólica ou auto-imune.

\section{PROTECÇÃO DE PESSOAS E ANIMAIS}

Os autores declaram que os procedimentos seguidos estavam de acordo com os regulamentos estabelecidos pelos responsáveis da Comissão de Investigação Clínica e Ética e de acordo com a Declaração de Helsínquia da Associação Médica Mundial.

\section{CONFIDENCIALIDADE DOS DADOS}

Os autores declaram ter seguido os protocolos do seu centro de trabalho acerca da publicação dos dados de doentes. 


\section{CONFLITO DE INTERESSES}

Não existem conflitos de interesse a declarar.

\section{REFERÊNCIAS}

1. Khaira HS, Rittoo D, Vohra RK, Smith SR. The non-ischaemic blue finger. Ann R Coll Surg Engl. 2001;83:154-7.

2. Cowen R, Richards T, Dharmadasa A, Handa A, Perkins JM. The acute blue finger: management and outcome. Ann R Coll Surg Engl. 2008;90:557-60.

3. Batra M, Tandon P, Gupta N. Clinical aproach to a patient with isolated digital ischaemia. JIACM. 2002;3:23-8.

\section{FONTES DE FINANCIAMENTO}

Não foi recebido qualquer subsídio ou bolsa.

4. Deliss LJ, Wilson JN. Acute blue fingers in women. J Bone Join Surg 1982;64:458-9

5. Harper C, Water P. Acute Idiopathic blue finger: Case report. J Hand Surg. 2013;38:1980-2.

6. Weinberg I, Jaff M. Spontaneus blue finger syndrome: a benign process Am J Med. 2012;125:e1-2.
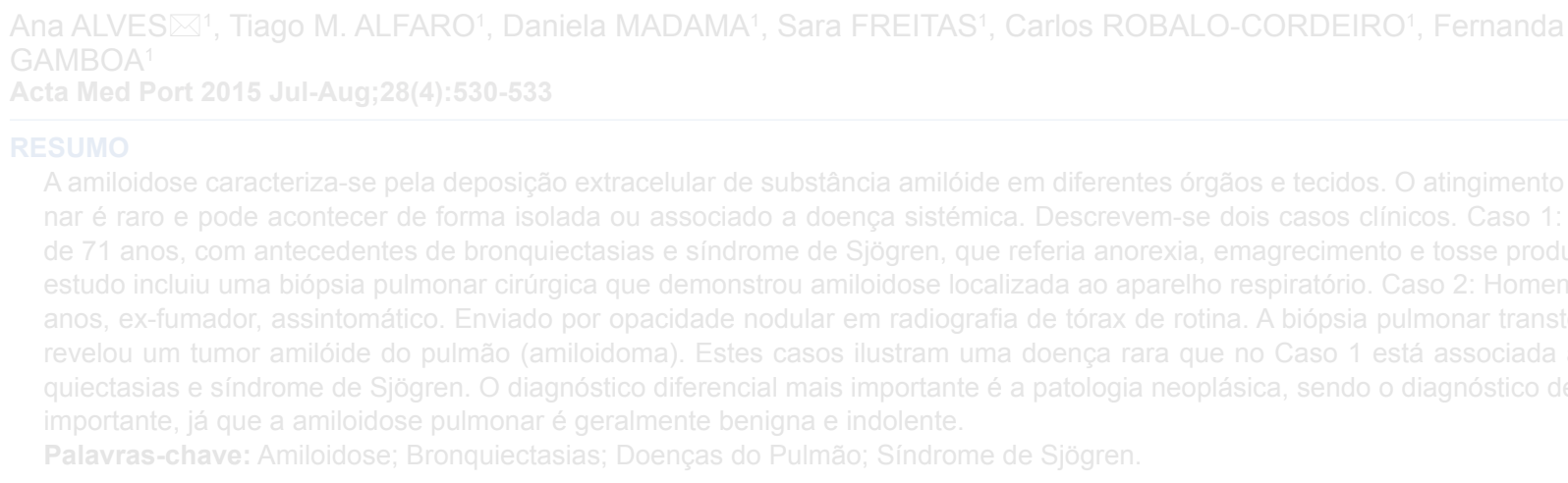

BSTRAC7

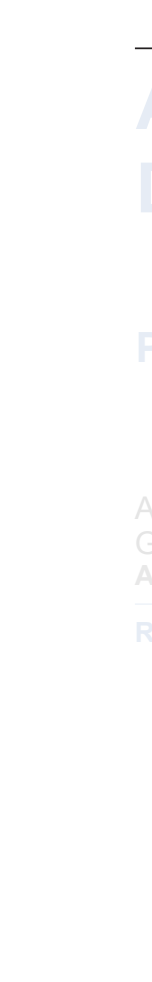




\section{Dedo Azul Não-Isquémico Espontâneo: Um Fenómeno Raro e Benigno}

Acta Med Port 2015:28:528-530

Publicado pela Acta Médica Portuguesa, a Revista Científica da Ordem dos Médicos

Av. Almirante Gago Coutinho, 151

1749-084 Lisboa, Portugal.

Tel: +351218428215

E-mail: submissao@actamedicaportuguesa.com

www.actamedicaportuguesa.com

ISSN:0870-399X | e-ISSN: 1646-0758

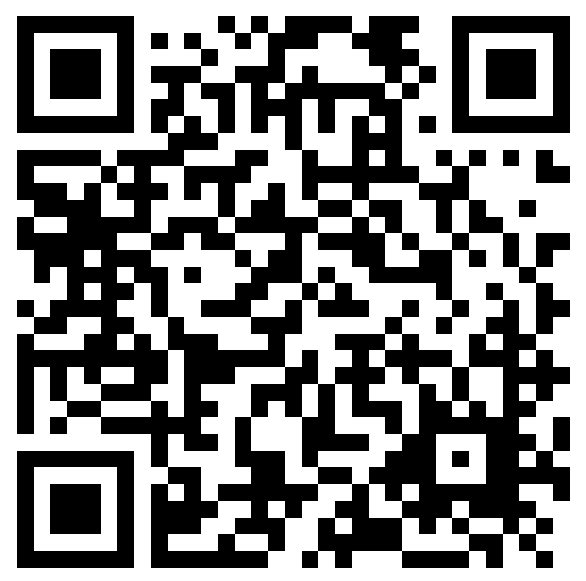

